Fecha de recepción: diciembre 2019 Fecha de aceptación: febrero 2020 Versión final: marzo 2020

\section{Craft your Future: diseñando desde la economía local, la artesanía y la tecnología}

Chele Esteve Sendra ${ }^{(1)}$, Manuel Martínez Torán ${ }^{(2)}$ y Ricardo Moreno Cuesta ${ }^{(3)}$

Resumen: El proyecto Craft Your Future une a instituciones educativas, estudiantes, autoridades locales, centros creativos y empresas (sociales) en la creación de una estrategia que ayude a las regiones a utilizar el patrimonio cultural intangible para aumentar su atractivo, impulsar las economías locales y construir un futuro basado en estos recursos regionales. La estrategia Craft Your Future se basa en la visión de que los jóvenes son la fuente de la economía del mañana y, por lo tanto, deben liderar el diseño de estrategias futuras. El desarrollo de las competencias de liderazgo de los estudiantes, la proactividad y la mentalidad empresarial conducirán el cambio y la innovación.

Palabras clave: Patrimonio cultural - Artesanía - Nuevas tecnologías - Sostenibilidad Dar forma a nuestras identidades y vidas - Economía circular - Formación.

[Resúmenes en inglés y portugués en las páginas 256-257]

(1) Chele Esteve Sendra es Profesor Senior Lecturer en la Universidad Politécnica de Valencia. Imparte Docencia en el Grado y Master de Diseño Industrial en la ETSID (Escuela Técnica Superior de Diseño Industrial) y en el Grado en Bellas Artes (UPV). Es Doctora "Cum Laude" Mención Internacional por la UPV (España) y Master of Arts (MA) Diseño Industrial por la University of Central England, Birmingham (Reino Unido). Actualmente participa como Investigador Principal en el proyecto titulado: Born alonge the Silk Road (Nacer en la ruta de la seda), auspiciado por de The International Institute for Central Asian Studies (IICAS), por UNESCO. Coordina el ETSID Desis Lab, Red Internacional de Diseño para la Sostenibilidad e Innovación Social. Desde 2010 Es Profesora visitante en el College of Design and Innovation de la Universidad de Tongji, Shanghai, China.

(2) Manuel Martínez Torán es Profesor Titular de la Universitat Politècnica de Valencia (UPV, España), imparte Docencia en el Grado de Diseño Industrial, en el Master en Tecnologías Interactivas y Fabricación Digital y en el Master Bussines Innovation, UPV. Investigador en el Centro de Gestión de la Calidad y del Cambio. Doctor por el Programa de Aproximaciones al Diseño Gráfico e Industrial de la UPV. Ha participado como Investigador Principal en más de 25 proyectos, con más de 90 publicaciones, 4 patentes y 9 tesis doctorales dirigidas. Director de la red FabLab Valencia y miembro de la Junta Directiva de la Red Española de Creación y Fabricación Digital, fue finalista de los Premios Nacionales de Artesanía en 2013. 
(3) Ricardo Moreno Cuesta es Profesor de Diseño de Producto e Investigador de Diseño en la Escuela de Arte y Superior de Diseño de Valencia. Fue Profesor de Dibujo en la Enseñanza Secundaria y Profesor de Formación Profesional, todos estos puestos mediante varias oposiciones aprobadas. Participa como Investigador en el Proyecto Born alonge the Silk Road (Nacer en la ruta de la seda), auspiciado por The International Institute for Central Asian Studies (IICAS) por UNESCO. Coordina el EASD Valencia Desis Lab, de la Red Internacional DESIS Network de Diseño para Sostenibilidad e Innovación Social. Sustain EASD, Valencia. Desde 2010 es Profesor visitante en el College of Design and Innovation de la Universidad de Tongji, Shanghai, China. En esta Universidad participó como Profesor invitado en Docencia de Cursos de Verano.

\section{Sobre el futuro de la artesanía}

Si nos situamos en el siglo XIX observamos como una patente facilitó el origen hacia una revolución, la industrial. Fue James Watt (1736-1819), su creador quién propulsó este cambio con la máquina de vapor que aplicó posteriormente a la locomotora y puso en marcha un avance tecnológico sin precedentes que marcó una nueva era. La transformación social que supuso todo ello se forjó ante una sociedad más liberal que buscaba aumentar la productividad de los recursos propios que conducirían sin duda hacia la economía y la eficiencia.

Por otro lado, en contra de la producción en masa, se erigió otro británico, William Morris (1834-1896), defendería la necesidad de crear con las manos objetos únicos, hermosos y asequibles. Fundador del movimiento 'Arts and Crafts', trabajaba por recuperar los métodos de producción propios de la Edad Media, trataba de capturar la historia y la tradición en piezas de diseño, en una defensa del oficio, el comercio justo y el valor de lo artesano frente a lo industrial.

En aquel momento ni Watt, ni Morris pudieron aunar su trabajo en una misma línea, aunque divergiera de algún modo, cualquier acercamiento era imposible, ni pensar que tecnología y artesanía pudiesen convivir en pro de un resultado común en beneficio de ambos. Morris fue la antítesis del progreso industrializado incluso utilizó la política como medio de defensa de sus ideales.

Veremos en el siglo XXI, y así observamos como dos siglos después la propuesta de convivencia entre artesanía y tecnología si puede funcionar y beneficiarse. En este sentido actualmente estamos trabajando e investigando sobre un Proyecto en el que reflexionamos y tratamos de implantar un modelo de trabajo para jóvenes, que se están formando en diferentes ramas del diseño.

Se apuesta por esta conciliación de la artesanía con los factores de estímulo que se forjan en sus vidas académicas:

1. su relación con la tecnología, y 
2. con todos los objetivos de la economía circular (reutilizar, reciclar, reducir, rediseñar y reparar).

Cuando hablamos de artesanía hablamos de un discurso contemporáneo, donde los jóvenes se interesen por las tradiciones, su significado y puedan dar una respuesta contextualizada culturalmente y también, encontrar la posibilidad de desarrollar sus iniciativas de empleo y emprendimiento.

Respecto a la tecnología, están aquellas que por ejemplo están permitiendo tener un encuentro entre las necesidades del productor y el estímulo que supone al joven poder acceder y recurrir a ellas. Para ello, la impresión 3D a modo de ejemplo, se postula como un recurso óptimo para conciliar tradición e innovación a través de la conexión entre los procesos digital y analógico.

Y finalmente, la economía circular como destino de las actividades que alrededor de estos dos procesos se encuentran, con el objetivo de centrar las fuentes de innovación que se originen por parte de jóvenes y estudiantes que participan en ellas, y como motivo para emprender y orientar sus esfuerzos hacia la creación de iniciativas circulares, o al menos, que estas ideas queden presentes en su quehacer diario.

Este Proyecto (ref. 2018-1-NL01-KA202-038952), está financiado por la Unión Europea a través del Programa K2 Erasmus+. El Proyecto vincula regiones con necesidades similares, pero involucra a socios con experiencia específica en los tres diferentes temas:

- Países Bajos (como expertos en economía circular),

- España (con experiencia en tecnologías de creación y fabricación digital) y

- Bulgaria (especializados en artesanías tradicionales).

\section{Caso de estudio: Craft your future}

En el marco del Programa Erasmus + KA2 Asociación Estratégica en Educación y Formación Profesional, se está llevando a cabo el proyecto Craft your future. El objetivo es mostrar el resultado de dos años de trabajo en las regiones de tres países como un estudio de caso, y que se pueda replicar esta experiencia en otros entornos de la Unión Europea. El uso sostenible del patrimonio cultural es esencial para garantizar que Leeuwarden (Países Bajos), Tryavna (Bulgaria) y Valencia (España), sigan siendo destinos populares para visitar y vivir. Las regiones participantes en Craft Your Future tienen gran arraigo en lo referente a tradiciones y artesanía tradicional. No obstante, existe una amenaza hacia las artesanías tradicionales que hace que tiendan a desaparecer gradualmente. Además, tenemos que observar como la sostenibilidad es uno de los principales desafíos sociales globales de nuestro tiempo. Se ha vuelto esencial crear una economía circular en la que se maximice la reutilización de productos y materias primas, y se minimice su destrucción de valor.

En el desarrollo del Proyecto, los estudiantes examinan por ejemplo si la tecnología y las tendencias avanzadas pueden ayudar a revitalizar las artesanías antiguas utilizando los desechos como materia prima. En el futuro, con una economía circular completa, el tér- 
mino 'desperdicio' será un concepto desactualizado. Nuestro objetivo ha sido colocar a los estudiantes a la cabeza del triángulo de la artesanía tradicional, las tendencias y las nuevas tecnologías, y la economía circular.

Craft Your Future, está dirigido principalmente a estudiantes en Universidades VET (educación y formación profesional). Los estudiantes de todos los campos de la educación participarán en el programa formativo desarrollado a partir de este conocimiento, creando un enfoque intersectorial y permitiendo que los estudiantes de diferentes orígenes aprendan unos de otros.

Los resultados que se han tomado para la evaluación del proyecto son:

- Desarrollar un programa de formación de estudiantes

- Crear un kit de herramientas fruto de la alianza regional

- Promover una estrategia regional con un plan de acción

- Tener una comunidad online

En cuanto a los socios, tres regiones han participado en el Proyecto Craft Your Future, lo que une a estas regiones es su fuerte tradición en artesanías tradicionales, su dependencia del patrimonio cultural como un factor económico importante y su ambición de educar a los estudiantes de Educación Superior (Formación Profesional-Técnica y Universitaria) para que tomen este tipo iniciativas.

- En los Países Bajos, los socios principales son Friesland College, House of Design y Learning Hub Friesland. Conviene señalar que también hay que incluir la colaboración de una empresa de tratamiento-reciclaje de residuos de Friesland, la Fundación Leeuwarden Cultural Capital of Europe 2018 y una asociación local de artesanías tradicionales.

- En España, intervienen dos socios, que cuentan con el respaldo de la Red FabLab VLC, la Escuela Técnica Superior del Diseño y el Centro de Artesanía de la Comunitat Valenciana, sitio donde además realizamos una primera exposición de resultados con los alumnos.

- En Bulgaria, los dos socios, cuentan con el apoyo del Museo Etnográfico de Gabrovo, donde se pudieron ver toda una representación de oficios tradicionales de la parte oriental del país.

La aportación específica de nuestro grupo, trata de medir los indicadores y el impacto que puede tener, sobre todo en jóvenes estudiantes de la Unión Europea. En este momento estamos evaluando el impacto y empezando a difundir estos logros.

Entre los más significativos conviene reseñar los datos obtenidos que indican la satisfacción de los jóvenes

- Donde consideran alto-muy alto el conocimiento que están obteniendo sobre las tradiciones y la artesanía de la Unión Europea. En este sentido destaca lo relevante que pueden suponer las mismas para su futuro.

- En cuanto al interés y las posibilidades de innovar a través de las tecnologías de fabricación digital (impresión 3D, corte láser y fresado $\mathrm{CNC}$ ) el dato es menor, pero con una cifra alta, aunque los jóvenes consideran que es muy importante para ellos. 
- Otras cifras relevantes que está dando cuenta el proyecto y que avanzaremos en futuras aportaciones, son el estímulo que ha supuesto creativamente para estos jóvenes participar en los encuentros que realizamos, y la relevancia de poder acceder a espacios que posibiliten esa creatividad durante y después de su formación académica, a través de makerspaces, fablabs o hubs creativos o de innovación.

No cabe duda que la alianza entre artesanía y tecnología va a tener un papel destacado en el siglo XXI.

\section{Bibliografía}

Andrews, M. \& Langmaid, R. (2003). The breakthrough zone: harnessing consumer creativity for business innovation. London: John Wiley \& Sons.

Bluet, J. C. \& Zemor, J. (1970). Prospective géographique: méthode et direction de récherches. Metra, vol. IX, 1.

Borja De Mozota, B. (2006). El diseño de la innovación, dos retos para la profesión del diseño. Temes de Disseny, 23. Barcelona: Elisava

Butler, J, S. \& Gibson, D. V. (2011). Global perspectives on technology transfer and commercialization: building innovative ecosystems. Camberley: Edward Elgar Publishing.

Etienne-Nugue, J. (2007). Háblame de la Artesanía. Col. Descubrir el Mundo, París: UNESCO, 2009.

FUTUR:E. (2010). The Next Best Brand. Seminario AEDEMO-AEA. Madrid: Observatorio Permanente de Tendencias.

Giddens, A.; Bauman, Z.; Luhmann, N.; Beck, U. \& Beriain, J. (comp.) (1996). Las consecuencias perversas de la modernidad: modernidad, contingencia y riesgo. Barcelona: Anthropos, 2007.

Gil, V. y Romero, F. (2008). Crossumer: claves para entender al consumidor español de nueva generación. Barcelona: Gestión 2000.

Godet, M. y Durance, P. (2011). La prospectiva estratégica para las empresas y los territorios. París: Dunod, UNESCO.

Goleman, D. (2009). Inteligencia ecológica. Barcelona: Kairós, 2010.

Gracht, H. A. (2008). The Future of Logistics: Scenarios for 2025. Col. Einkauf, Logistik und Supply Chain Management. Wiesbaden: Gabler Verlag.

Hamermesh, R. G. (1990). Planeación estratégica. O como se las arreglan los gerentes triunfadores. México. Noriega Limusa.

Hatchuel, A. \& Weil, B. (2002). C-K theory: Notions and applications of a unified design theory. Proceedings of the Herbert Simon International Conference on Design Sciences. Lyon, 15-16 March.

Hill, C. W. L. y Jones, G. R. (1996). Administración estratégica: Un enfoque integrado. New York: Mc Graw Hill.

Hofer, W. C. y Schendel, D. (1978). Strategy formulation: Analytical concepts. Minnesota: West Publishing. 
Kalthoff, O.; Nonaka, I. y Nueno, P. (1998). La Luz y la Sombra. La innovación en la empresa y sus formas de gestión. Bilbao: Ediciones Deusto.

Kotler, P. y Keller, K. L. (2006). Dirección de Marketing. Valencia: Pearson Educación, 2009. Lehni, M. (2000). Ecoeficiencia: Creando más valor con menos impacto. Free Translate by CECODES, Colombia. World Business Council for Sustainable Development, UK.

Levine, R. (et al.) (2000). El manifiesto Cluetrain: el ocaso de la empresa convencional. Barcelona: Deusto, 2008.

Linstone, H. \& Turoff, M. (1975). The Delphi Method. Techniques and applications. Utah: Addison-Wesley.

Lorange, P. (1980). Corporate planning. New Jersey: Prentice Hall.

Macafee, A. (2009). Enterprise 2.0: New collaborative tools for your organization's toughest challenges. Harvard: Harvard Business Publishing.

Navinchandra, D. (1990). Innovative Design System: Where are we, and where do we go from here? Technical Report CMU-RI-TR-90-01. The Robotic Institute. Carnegie Mellon University. Pittsburgh, 12 January.

Newman, W. H. \& Logan, J. P. (1976). Strategy, policy and central management. Mason: South - Western.

Nombela, G. (coord.) (2010). Ideas para una nueva economía: hacia una España más sostenible en 2025. Madrid: Fundación IDEAS.

Nii, A. A.; Emmanuel, M, \& Colin, B. Cradle to Cradle Implementation in Business Sites and the Perspectives of Tenant Stakeholders. Energy Procedia 2015, 83, 31-40. DOI: 10.1016/j. egypro.2015.12.193.

Peppers D.; Rogers, M. \& Dorf, B. (1999). The one to one fieldbook: the complete toolkit for implementing a 1 to 1 marketing program. New York: Currency Doubleday.

Porter, M. (1993). Estrategia competitiva. Técnicas de análisis de los sectores industriales y de la competencia. México: Compañía Editorial Continental.

Prahalad, C. K. y Ramaswamy, V. (2003). El futuro de la competencia: creación conjunta de valor único con los consumidores. Barcelona: Gestión 2000, 2004.

Rodríguez Ferrándiz, R. (2010). La musa venal: producción y consumo de la cultura industrial. Murcia: Tres Fronteras.

Schank, R. (2011). Teaching Minds: How Cognitive Science Can Save Our Schools. New York: Teachers College Press.

Tapscott, D. y Williams, A. (2006). Wikinomics: la nueva economía de las multitudes inteligentes. Barcelona: Paidós, 2009.

Wokstrom, O. (2001). El elogio de la lentitud. Barcelona: Editorial Norma, 2006.

Abstract: The Craft Your Future project brings together educational institutions, students,
local authorities, creative centers and (social) companies in the creation of a strategy that
helps regions use intangible cultural heritage to increase their attractiveness, boost local
economies and build a future based on these regional resources. The Craft Your Future
strategy is based on the vision that young people are the source of tomorrow's economy 
and, therefore, should lead the design of future strategies. The development of student leadership skills, proactivity and business mentality will drive change and innovation.

Keywords: Cultural heritage - Craftsmanship - New technologies - Sustainability - Shaping our identities and lives - Circular economy - Training.

Resumo: O projeto Craft Your Future reúne instituições educacionais, estudantes, autoridades locais, centros criativos e empresas (sociais) na criação de uma estratégia que ajude as regiões a usar o patrimônio cultural intangível para aumentar sua atratividade, impulsionar as economias locais e construir um futuro com base nesses recursos regionais. A estratégia Craft Your Future baseia-se na visão de que os jovens são a fonte da economia de amanhã e, portanto, devem liderar o desenho de estratégias futuras. O desenvolvimento de habilidades de liderança estudantil, proatividade e mentalidade comercial promoverão mudanças e inovação.

Palavras chave: Patrimônio cultural - Artesanato - Novas tecnologias - Sustentabilidade Moldando nossas identidades e vidas - Economia circular - Treinamento. 